\title{
Copula Representations for Invariant Dependence Functions
}

\author{
Jayme Pinto and Nikolai Kolev
}

\begin{abstract}
Our main goal is to characterize in terms of copulas the linear Sibuya bivariate lack of memory property recently introduced in [12]. As a particular case, one can obtain nonaging copulas considered in the literature.
\end{abstract}

\section{Introduction and Preliminaries}

Let $X_{i}$ be non-negative continuous random variables with survival functions $S_{X_{i}}\left(x_{i}\right)$ $=P\left(X_{i}>x_{i}\right)$ and densities $f_{X_{i}}\left(x_{i}\right), i=1,2$. Denote by $S\left(x_{1}, x_{2}\right)=P\left(X_{1}>\right.$ $\left.x_{1}, X_{2}>x_{2}\right)$, the joint survival function of the random vector $\left(X_{1}, X_{2}\right)$. Following [13], any bivariate survival function can be decomposed as a product of marginal survival functions and a dependence function $\Omega\left(x_{1}, x_{2}\right)$ via

$$
S\left(x_{1}, x_{2}\right)=S_{X_{1}}\left(x_{1}\right) S_{X_{2}}\left(x_{2}\right) \Omega\left(x_{1}, x_{2}\right) \text { for all } x_{1}, x_{2} \geq 0 \text {. }
$$

The function $\Omega\left(x_{1}, x_{2}\right)$ represents the free-of-margin influence contribution to the genuine dependence advocated by $S\left(x_{1}, x_{2}\right)$. A family of Sibuya copulas is introduced in [6], where the authors are motivated by a particular dynamic default model.

Our analysis is based on the following relation

$$
S\left(x_{1}+t, x_{2}+t\right)=S\left(x_{1}, x_{2}\right) S(t, t) B\left(x_{1}, x_{2} ; t\right), \quad t>0
$$

where $B\left(x_{1}, x_{2} ; t\right)$ is an appropriate "aging" function satisfying the boundary conditions $B\left(x_{1}, x_{2} ; 0\right)=B(0,0 ; t)=1$. In fact, incorporating a time component in the arguments, we replace the product of marginal survival functions in (1) by the product of joint survival functions with nonoverlapping arguments.

\footnotetext{
J. Pinto $(\varangle) \cdot$ N. Kolev

Department of Statistics, University of São Paulo, São Paulo, Brazil

e-mail: jaymeaugusto@gmail.com

N. Kolev

e-mail: kolev.ime@gmail.com

$\mathrm{K}$. Glau et al. (eds.), Innovations in Quantitative Risk Management, Springer Proceedings in Mathematics \& Statistics 99, 
In the simplest case, when $B\left(x_{1}, x_{2} ; t\right)=1$ in (2), one gets the functional equation

$$
S\left(x_{1}+t, x_{2}+t\right)=S\left(x_{1}, x_{2}\right) S(t, t)
$$

for all $x_{1}, x_{2} \geq 0$ and $t>0$. Bivariate continuous distributions satisfying (3) possess the classical bivariate lack of memory property (BLMP).

The only solution of (3) with exponential marginals is the Marshall-Olkin bivariate exponential distribution introduced in [9]. However, there do exist distributions having BLMP with nonexponential marginals. Various solutions of functional equation (3) are presented in [7] where the marginals may have any kind of failure rates: increasing, decreasing, bathtub, etc. It is well-known that BLMP preserves the distribution of $\left(X_{1}, X_{2}\right)$ and its residual lifetime vector

$$
\mathbf{X}_{t}=\left(X_{1 t}, X_{2 t}\right)=\left[\left(X_{1}-t, X_{2}-t\right) \mid X_{1}>t, X_{2}>t\right]
$$

independent of $t \geq 0$, i.e., $\left(X_{1}, X_{2}\right) \stackrel{d}{=} \mathbf{X}_{t}$ implying $X_{i} \stackrel{d}{=} X_{i t}, i=1,2$ for all $t \geq 0$.

Remark 1 The vectors $\left(X_{1}, X_{2}\right)$ and $\mathbf{X}_{t}$ should necessarily have the same survival copula, which is unique under continuity of $X_{i}, i=1,2$. Therefore, BLMP implies that the corresponding survival copulas are time invariant (nonaging).

The joint survival function of $\mathbf{X}_{t}$ is given by $S_{\mathbf{X}_{t}}\left(x_{1}, x_{2}\right)=S\left(x_{1}+t, x_{2}+t\right) / S(t, t)$. Its marginal survival functions are $S_{X_{1 t}}\left(x_{1}\right)=S\left(x_{1}+t, t\right) / S(t, t)$ and $S_{X_{2 t}}\left(x_{2}\right)=$ $S\left(t, x_{2}+t\right) / S(t, t)$. Applying the Sibuya form representation (1) with respect to the residual lifetime vector $\mathbf{X}_{t}$ we have

$$
S_{\mathbf{X}_{t}}\left(x_{1}, x_{2}\right)=S_{X_{1 t}}\left(x_{1}\right) S_{X_{2 t}}\left(x_{2}\right) \Omega_{t}\left(x_{1}, x_{2}\right)
$$

where $\Omega_{t}\left(x_{1}, x_{2}\right)$ is the dependence function of $\mathbf{X}_{t}$.

We will consider a class of continuous bivariate distributions preserving $\Omega_{t}\left(x_{1}, x_{2}\right)$ independent of $t \geq 0$, i.e., imposing condition $\Omega_{t}\left(x_{1}, x_{2}\right)=\Omega\left(x_{1}, x_{2}\right)$, where $\Omega\left(x_{1}, x_{2}\right)$ is the dependence function of $\left(X_{1}, X_{2}\right)$ from (1). Such a class with memoryless dependence function has been recently introduced in [12] as follows.

Definition 1 The nonnegative continuous bivariate distribution $\left(X_{1}, X_{2}\right)$ possesses linear Sibuya BLMP (to be abbreviated LS-BLMP) if

$$
\frac{S_{\mathbf{X}_{t}}\left(x_{1}, x_{2}\right)}{S_{X_{1 t}}\left(x_{1}\right) S_{X_{2 t}}\left(x_{2}\right)}=\frac{S\left(x_{1}, x_{2}\right)}{S_{X_{1}}\left(x_{1}\right) S_{X_{2}}\left(x_{2}\right)}
$$

for all $x_{1}, x_{2}, t \geq 0$ and

$$
S_{X_{i t}}\left(x_{i}\right)=S_{X_{i}}\left(x_{i}\right) \exp \left\{-a_{i} x_{i} t\right\} \text { for } a_{i} \geq 0, i=1,2 .
$$


Observe that BLMP distributions satisfy (5). This means that the class of bivariate continuous distributions with LS-BLMP includes those possessing BLMP.

Let us assume that the partial derivatives of $S\left(x_{1}, x_{2}\right)$ exist and are continuous. Denote by $r_{i}\left(x_{1}, x_{2}\right)=-\partial \ln S\left(x_{1}, x_{2}\right) / \partial x_{i}$ the conditional failure rates, $i=1,2$. In [12] it is introduced a class $\mathscr{L}(\mathbf{x} ; \mathbf{a})$ of nonnegative bivariate continuous distributions that satisfy the relation

$$
r\left(x_{1}, x_{2}\right)=r_{1}\left(x_{1}, x_{2}\right)+r_{2}\left(x_{1}, x_{2}\right)=a_{0}+a_{1} x_{1}+a_{2} x_{2} \text { for } a_{0}, a_{1}, a_{2} \geq 0
$$

for all $x_{1}, x_{2} \geq 0$, where $\mathbf{x}=\left(x_{1}, x_{2}\right)$ and $\mathbf{a}=\left(a_{0}, a_{1}, a_{2}\right)$ is the parameter vector.

When the survival function $S\left(x_{1}, x_{2}\right)$ is differentiable, the sum $r_{1}\left(x_{1}, x_{2}\right)+$ $r_{2}\left(x_{1}, x_{2}\right)$ has the following interpretation in terms of directional derivatives: it establishes the performance of $-\ln \left[S\left(x_{1}, x_{2}\right)\right]$ along the lines parallel to $\left\{x_{1}=x_{2}\right\}$, i.e., with $45^{\circ}$ inclination.

Managing a portfolio means observing and controlling its value changes over time to achieve a desired outcome. The vector $\left(r_{1}\left(x_{1}, x_{2}\right), r_{2}\left(x_{1}, x_{2}\right)\right)$ of partial derivatives of $-\ln \left[S\left(x_{1}, x_{2}\right)\right]$ is its gradient. With the gradient at hand, the risk manager can evaluate the incremental impact of changes to the portfolio.

The Marshall-Olkin bivariate exponential distribution is a widely used model in risk management and possesses BLMP, see Chap. 3 in [8]. The class $\mathscr{L}(\mathbf{x} ; \mathbf{a})$ transforms into BLMP when $a_{1}=a_{2}=0$ in (7) and $r_{1}\left(x_{1}, x_{2}\right)+r_{2}\left(x_{1}, x_{2}\right)=a_{0}$.

The sum in (7) may serve as a complementary risk measure. For example, the portfolio can be considered "risky" if $r_{1}\left(x_{1}, x_{2}\right)+r_{2}\left(x_{1}, x_{2}\right)>a_{0}+a_{1} x_{1}+a_{2} x_{2}$, where parameters $a_{0}, a_{1}$ and $a_{2}$ are preliminary fixed by an expert.

The joint survival function corresponding to (7) is given by

$$
S\left(x_{1}, x_{2}\right)= \begin{cases}S_{X_{1}}\left(x_{1}-x_{2}\right) \exp \left\{-a_{0} x_{2}-a_{1} x_{1} x_{2}-\frac{a_{2}-a_{1}}{2} x_{2}^{2}\right\}, & \text { if } x_{1} \geq x_{2} \geq 0 \\ S_{X_{2}}\left(x_{2}-x_{1}\right) \exp \left\{-a_{0} x_{1}-a_{2} x_{1} x_{2}-\frac{a_{1}-a_{2}}{2} x_{1}^{2}\right\}, & \text { if } x_{2} \geq x_{1} \geq 0\end{cases}
$$

Remark 2 The joint survival function $S\left(x_{1}, x_{2}\right)$ in the previous expression is proper only for certain marginals $S_{X_{1}}\left(x_{1}\right)$ and $S_{X_{2}}\left(x_{2}\right)$. Their choice will determine the range of possible values for the non-negative parameters $a_{0}, a_{1}$ and $a_{2}$, see Theorem 5.2.14 and Proposition 5.2.17 in [12]. The nonnegative parameter $a_{0}$ plays an important role in the class $\mathscr{L}(\mathbf{x} ; \mathbf{a})$. If $a_{0}=f_{X_{1}}(0)+f_{X_{2}}(0)$, the joint survival function $S\left(x_{1}, x_{2}\right)$ is absolutely continuous and if $a_{0}<f_{X_{1}}(0)+f_{X_{2}}(0)$, the distribution exhibits a singular component.

It happens that the class $\mathscr{L}(\mathbf{x} ; \mathbf{a})$ specified by (7) can be characterized by the LS-BLMP defined by (5) and (6). The class $\mathscr{L}(\mathbf{x} ; \mathbf{a})$ contains continuous bivariate distributions that are symmetric or asymmetric, positive quadrant dependent or negative quadrant dependent, absolutely continuous or exhibit a singular component. In addition, $\mathscr{L}(\mathbf{x} ; \mathbf{a})$ can be equivalently represented by relation (2) when $B\left(x_{1}, x_{2} ; t\right)=\exp \left\{-a_{1} x_{1} t-a_{2} x_{2} t\right\}$, i.e., by 


$$
\frac{S\left(x_{1}+t, x_{2}+t\right)}{S(t, t)}=S\left(x_{1}, x_{2}\right) \exp \left\{-a_{1} x_{1} t-a_{2} x_{2} t\right\} .
$$

In Sect. 2, we will characterize the class $\mathscr{L}(\mathbf{x} ; \mathbf{a})$ (or equivalently LS-BLMP) in copula terms using the functional equation (8) as base. Recall that the time invariance (nonaging) phenomena of the dependence function $\Omega\left(x_{1}, x_{2}\right)$ concerns the preservation of the dependence function $\Omega_{t}\left(x_{1}, x_{2}\right)$ given in Sibuya form (4). This justifies our suggestion to the corresponding copula be named "Sibuya-type copula." In Sect. 3, we discuss bivariate survival functions with nonaging survival copulas and obtain known relations as particular cases of our findings.

\section{Copula Representations of the Class $\mathscr{L}(\mathbf{x} ; \mathbf{a})$}

Let the vector $\left(X_{1}, X_{2}\right)$ be a member of the class $\mathscr{L}(\mathbf{x} ; \mathbf{a})$. Hence, the survival function of the corresponding residual lifetime vector $\mathbf{X}_{t}$ is given by (8). Denote by $C$ and $C_{t}$, the survival copulas of $\left(X_{1}, X_{2}\right)$ and $\mathbf{X}_{t}$, respectively. First, we will find a relation between the survival copulas $C$ and $C_{t}$. As a second step, we will obtain a characterizing functional equation for the survival copula $C_{t}$ that joins the corresponding marginals in both sides of (8).

Theorem 1 Let $\left(X_{1}, X_{2}\right)$ belong to the class $\mathscr{L}(\mathbf{x} ; \mathbf{a})$. The survival copulas of $\mathbf{X}_{t}$ and $\left(X_{1}, X_{2}\right)$ are connected by

$$
\begin{aligned}
C_{t}(u, v)= & C\left(\exp \left\{-H_{1}\left(G_{1 t}^{-1}(-\ln u)\right)\right\}, \exp \left\{-H_{2}\left(G_{2 t}^{-1}(-\ln v)\right)\right\}\right) \\
& \times \exp \left\{-a_{1} t G_{1 t}^{-1}(-\ln u)-a_{2} t G_{2 t}^{-1}(-\ln v)\right\},
\end{aligned}
$$

where $u, v \in(0,1], H_{i}\left(x_{i}\right)=-\ln \left[S_{X_{i}}\left(x_{i}\right)\right]$ and $G_{i t}\left(x_{i}\right)=H_{i}\left(x_{i}\right)+a_{i} x_{i} t, i=1,2$.

Proof The marginals of $\mathbf{X}_{t}$ have survival functions specified by (6). Using Sklar's theorem, relation (8) can be rewritten in terms of the survival copulas $C_{t}$ and $C$ as follows

$$
\begin{gathered}
C_{t}\left(S_{X_{1}}\left(x_{1}\right) \exp \left\{-a_{1} x_{1} t\right\}, S_{X_{2}}\left(x_{2}\right) \exp \left\{-a_{2} x_{2} t\right\}\right) \\
=C\left(S_{X_{1}}\left(x_{1}\right), S_{X_{2}}\left(x_{2}\right)\right) \exp \left\{-a_{1} x_{1} t-a_{2} x_{2} t\right\} .
\end{gathered}
$$

Let $u=S_{X_{1}}\left(x_{1}\right) \exp \left\{-a_{1} x_{1} t\right\}$ and $v=S_{X_{2}}\left(x_{2}\right) \exp \left\{-a_{2} x_{2} t\right\}$. From the relations $S_{X_{i}}\left(x_{i}\right)=\exp \left\{-H_{i}\left(x_{i}\right)\right\}$ and $G_{i t}\left(x_{i}\right)=H_{i}\left(x_{i}\right)+a_{i} x_{i} t, i=1,2$, we get $x_{1}=$ $G_{1 t}^{-1}(-\ln u)$ and $x_{2}=G_{2 t}^{-1}(-\ln v)$. Using these Eqs. in (10) we obtain (9).

Relation (9) shows that the survival copulas of $\left(X_{1}, X_{2}\right)$ and $\mathbf{X}_{t}$ do not coincide in general. The time invariance (nonaging) in the class $\mathscr{L}(\mathbf{x} ; \mathbf{a})$ (being equivalent to LS$\mathrm{BLMP}$ ) is related to the memoryless dependence function $\Omega_{t}$ of the residual lifetime 
vector $\mathbf{X}_{t}$, see relation (5). For comparison only, recall that the time invariance for BLMP distributions is concerned with the joint distribution of $\mathbf{X}_{t}$.

Substituting $a_{1}=a_{2}=0$ in (9), we get $C_{t}(u, v)=C(u, v)$ for all $t \geq 0$, i.e., the survival copula $C_{t}$ is time invariant, see Remark 1 . The conclusion is same if $X_{1}$ and $X_{2}$ are independent, i.e., $C(u, v)=u v$. Thus, we have the following result.

Corollary 1 Under conditions of Theorem 1 if

(i) $a_{1}=a_{2}=0$ or

(ii) $X_{1}$ is independent of $X_{2}$,

then $C_{t}(u, v)=C(u, v)$ for all $u, v \in(0,1]$ and $t \geq 0$.

The next example illustrates the relations established.

Example 1 Let the vector $\left(X_{1}, X_{2}\right)$ belong to $\mathscr{L}(\mathbf{x} ; \mathbf{a})$. Suppose that the marginals are exponentially distributed, i.e., $S_{X_{i}}(x)=\exp \left\{-\lambda_{i} x_{i}\right\}, \lambda_{i}>0, i=1,2$. Therefore, $G_{i t}(x)=\lambda_{i} x+a_{i} x t$ and $G_{i t}^{-1}(u)=u /\left(\lambda_{i}+a_{i} t\right), i=1$, 2. From (9) we obtain

$$
C_{t}(u, v)=C\left(\exp \left\{\frac{\lambda_{1} \ln u}{\lambda_{1}+a_{1} t}\right\}, \exp \left\{\frac{\lambda_{2} \ln v}{\lambda_{2}+a_{2} t}\right\}\right) \exp \left\{\frac{a_{1} t \ln u}{\lambda_{1}+a_{1} t}+\frac{a_{2} t \ln v}{\lambda_{2}+a_{2} t}\right\}
$$

which can be simplified to

$$
C_{t}(u, v)=C\left(u^{\frac{\lambda_{1}}{\lambda_{1}+a_{1} t}}, v^{\frac{\lambda_{2}}{\lambda_{2}+a_{2} t}}\right) u^{\frac{a_{1} t}{\lambda_{1}+a_{1} t}} v^{\frac{a_{2} t}{\lambda_{2}+a_{2} t}}
$$

Relation (11) gives a general expression for the survival copula $C_{t}(u, v)$ corresponding to $\mathbf{X}_{t}$ for all members of the class $\mathscr{L}(\mathbf{x} ; \mathbf{a})$ with exponential marginals.

Assume further that $\left(X_{1}, X_{2}\right)$ follows Gumbel's type I exponential distribution with survival function

$$
S\left(x_{1}, x_{2}\right)=\exp \left\{-\lambda_{1} x_{1}-\lambda_{2} x_{2}-\boldsymbol{\theta} \lambda_{1} \lambda_{2} x_{1} x_{2}\right\}, \quad \boldsymbol{\theta} \in[0,1], \lambda_{1}, \lambda_{2}>0,
$$

see [5]. This distribution is a member of the class $\mathscr{L}(\mathbf{x} ; \mathbf{a})$ and the constants in (7) are specified by $a_{0}=\lambda_{1}+\lambda_{2}$ and $a_{1}=a_{2}=\boldsymbol{\theta} \lambda_{1} \lambda_{2}$. The corresponding survival copula is $C(u, v)=u v \exp \{-\boldsymbol{\theta} \ln u \ln v\}$. Substituting $C(u, v)$ in (11) we obtain $C_{t}(u, v)=u v \exp \left\{-\boldsymbol{\theta} \ln u \ln v /\left[\left(1+\boldsymbol{\theta} \lambda_{2} t\right)\left(1+\boldsymbol{\theta} \lambda_{1} t\right)\right]\right\}$. Therefore, the survival copula $C_{t}(u, v)$ depends on $t$ as well.

When $t=0$ in (11) we recover the survival copula $C(u, v)$ of $\left(X_{1}, X_{2}\right)$ and letting $t \rightarrow \infty$, we obtain the independence copula $C_{\infty}(u, v)=u v$. Notice that the independence of $X_{1}$ and $X_{2}$ is equivalent to the condition $a_{1}=a_{2}=0$.

Now, our interest is to find a characterizing functional equation involving the survival copula $C_{t}$ of $\mathbf{X}_{t}$ for the absolutely continuous members of the class $\mathscr{L}(\mathbf{x} ; \mathbf{a})$.

Theorem 2 Let the survival copula $C_{t}$ of $\mathbf{X}_{t}$ be differentiable in its arguments. The absolutely continuous random vector $\left(X_{1}, X_{2}\right)$ belongs to the class $\mathscr{L}(\mathbf{x} ; \mathbf{a})$, if and only if there exist non-negative constants $a_{1}$ and $a_{2}$, such that 


$$
C_{t}\left(\frac{S\left(x_{1}+t, t\right)}{S(t, t)}, \frac{S\left(t, x_{2}+t\right)}{S(t, t)}\right)=C_{t}\left(S_{X_{1}}\left(x_{1}\right) \exp \left\{-a_{1} x_{1} t\right\}, S_{X_{2}}\left(x_{2}\right) \exp \left\{-a_{2} x_{2} t\right\}\right),
$$

for all $x_{1}, x_{2}, t \geq 0$.

Proof Let us assume that the functional equation(12) is satisfied. We will show that (7) is fulfilled. Taking the derivative in both sides of (12) with respect to $t$ we obtain

$$
\begin{aligned}
& C_{t}^{1}\left(\frac{S\left(x_{1}+t, t\right)}{S(t, t)}, \frac{S\left(t, x_{2}+t\right)}{S(t, t)}\right) \frac{\left[S^{1}\left(x_{1}+t, t\right)+S^{2}\left(x_{1}+t, t\right)\right] S(t, t)-S\left(x_{1}+t, t\right)\left[S^{1}(t, t)+S^{2}(t, t)\right]}{[S(t, t)]^{2}} \\
& \quad+C_{t}^{2}\left(\frac{S\left(x_{1}+t, t\right)}{S(t, t)}, \frac{S\left(t, x_{2}+t\right)}{S(t, t)}\right) \frac{\left[S^{1}\left(t, x_{2}+t\right)+S^{2}\left(t, x_{2}+t\right)\right] S(t, t)-S\left(t, x_{2}+t\right)\left[S^{1}(t, t)+S^{2}(t, t)\right]}{[S(t, t)]^{2}} \\
& \quad=C_{t}^{1}\left(S_{X_{1}}\left(x_{1}\right) \exp \left\{-a_{1} x_{1} t\right\}, S_{X_{2}}\left(x_{2}\right) \exp \left\{-a_{2} x_{2} t\right\}\right)\left(-a_{1} x_{1} S_{X_{1}}\left(x_{1}\right) \exp \left\{-a_{1} x_{1} t\right\}\right) \\
& \quad+C_{t}^{2}\left(S_{X_{1}}\left(x_{1}\right) \exp \left\{-a_{1} x_{1} t\right\}, S_{X_{2}}\left(x_{2}\right) \exp \left\{-a_{2} x_{2} t\right\}\right)\left(-a_{2} x_{2} S_{X_{2}}\left(x_{2}\right) \exp \left\{-a_{2} x_{2} t\right\}\right),
\end{aligned}
$$

where the superscripts ${ }^{1}$ and ${ }^{2}$ denote the partial derivatives with respect to the first and second arguments of the corresponding functions. Letting $x_{1}=0$ in the last equation we have

$$
\begin{aligned}
C_{t}^{2} & \left(1, \frac{S\left(t, x_{2}+t\right)}{S(t, t)}\right) \frac{\left[S^{1}\left(t, x_{2}+t\right)+S^{2}\left(t, x_{2}+t\right)\right] S(t, t)-S\left(t, x_{2}+t\right)\left[S^{1}(t, t)+S^{2}(t, t)\right]}{[S(t, t)]^{2}} \\
& =C_{t}^{2}\left(1, S_{X_{2}}\left(x_{2}\right) \exp \left\{-a_{2} x_{2} t\right\}\right)\left(-a_{2} x_{2} S_{X_{2}}\left(x_{2}\right) \exp \left\{-a_{2} x_{2} t\right\}\right)
\end{aligned}
$$

When $x_{i}=0$ in (12) we get relations (6) in Definition $1, i=1,2$ and therefore

$$
\frac{\left[S^{1}\left(t, x_{2}+t\right)+S^{2}\left(t, x_{2}+t\right)\right] S(t, t)-S\left(t, x_{2}+t\right)\left[S^{1}(t, t)+S^{2}(t, t)\right]}{[S(t, t)]^{2}}=-a_{2} x_{2} S_{X_{2}}\left(x_{2}\right) \exp \left\{-a_{2} x_{2} t\right\} .
$$

Since $r\left(t, x_{2}+t\right)=-\left[S^{1}\left(t, x_{2}+t\right)+S^{2}\left(t, x_{2}+t\right)\right] / S\left(t, x_{2}+t\right)$ and $r(t, t)=\left[S^{1}(t, t)+S^{2}(t, t)\right] / S(t, t)$ we get

$$
-\frac{S\left(t, x_{2}+t\right)}{S(t, t)}\left[r\left(t, x_{2}+t\right)-r(t, t)\right]=-a_{2} x_{2} S_{X_{2}}\left(x_{2}\right) \exp \left\{-a_{2} x_{2} t\right\},
$$

which is equivalent to

$$
r\left(t, x_{2}+t\right)=r(t, t)+a_{2} x_{2}
$$

Analogously we obtain the equation

$$
r\left(x_{1}+t, t\right)=r(t, t)+a_{1} x_{1} .
$$

Now, we will represent $r(t, t)$ as a function of $a_{0}, a_{1}, a_{2}$ and $t$. Taking the partial derivative of (12) with respect to $x_{1}$ we have

$$
\begin{aligned}
C_{t}^{1}\left(\frac{S\left(x_{1}+t, t\right)}{S(t, t)}, \frac{S\left(t, x_{2}+t\right)}{S(t, t)}\right) \frac{S^{1}\left(x_{1}+t, t\right)}{S(t, t)}= & C_{t}^{1}\left(S_{X_{1}}\left(x_{1}\right) \exp \left\{-a_{1} x_{1} t\right\}, S_{X_{2}}\left(x_{2}\right) \exp \left\{-a_{2} x_{2} t\right\}\right) \\
& \times\left(-f_{X_{1}}\left(x_{1}\right) \exp \left\{-a_{1} x_{1} t\right\}-a_{1} t S_{X_{1}}\left(x_{1}\right) \exp \left\{-a_{1} x_{1} t\right\}\right) .
\end{aligned}
$$


Applying (6) in the last equation we obtain

$$
\frac{S^{1}\left(x_{1}+t, t\right)}{S(t, t)}=-f_{X_{1}}\left(x_{1}\right) \exp \left\{-a_{1} x_{1} t\right\}-a_{1} t S_{X_{1}}\left(x_{1}\right) \exp \left\{-a_{1} x_{1} t\right\}
$$

and putting $x_{1}=0$ we have $r_{1}(t, t)=f_{X_{1}}(0)+a_{1} t$. Similarly we get $r_{2}(t, t)=$ $f_{X_{2}}(0)+a_{2} t$. The sum of last two equations gives

$$
r(t, t)=r_{1}(t, t)+r_{2}(t, t)=\left[f_{X_{1}}(0)+f_{X_{2}}(0)\right]+a_{1} t+a_{2} t .
$$

Let $t=0$ in last relation to get $f_{X_{1}}(0)+f_{X_{2}}(0)=a_{0} \geq 0$. Thus,

$$
r(t, t)=a_{0}+a_{1} t+a_{2} t
$$

Taking into account (13) and (14), we conclude that $r\left(x_{1}, x_{2}\right)=a_{0}+a_{1} x_{1}+a_{2} x_{2}$. Therefore, we obtain the relation (7) which defines the class $\mathscr{L}(\mathbf{x} ; \mathbf{a})$. In addition, the corresponding bivariate distributions are absolutely continuous because of equation $f_{X_{1}}(0)+f_{X_{2}}(0)=a_{0}$, see Remark 2 .

Conversely, assume that the random vector $\left(X_{1}, X_{2}\right)$ belonging to the class $\mathscr{L}(\mathbf{x} ; \mathbf{a})$ is absolutely continuous. Therefore (8), being equivalent to (5) and (6), is valid. In addition, relations (6) show that the marginal distributions in both sides of (8) coincide. Applying Sklar's theorem to (8), we obtain the functional equation(12).

Since the dependence function $\Omega_{t}$ satisfies the Sibuya form (4), we refer to the survival copula $C_{t}$ characterized by functional equation(12) as Sibuya-type copula.

Example 2 Let us consider the absolutely continuous joint survival function

$$
S\left(x_{1}, x_{2}\right)= \begin{cases}\exp \left\{-\left[\lambda_{1} x_{1}+\lambda_{2} x_{2}+\lambda_{1} \lambda_{2} x_{2}\left(\boldsymbol{\theta}_{1} x_{1}+\frac{\boldsymbol{\theta}_{2}-\boldsymbol{\theta}_{1}}{2} x_{2}\right)\right]\right\}, & \text { if } x_{1} \geq x_{2} \geq 0 \\ \exp \left\{-\left[\lambda_{1} x_{1}+\lambda_{2} x_{2}+\lambda_{1} \lambda_{2} x_{1}\left(\boldsymbol{\theta}_{2} x_{2}+\frac{\boldsymbol{\theta}_{1}-\boldsymbol{\theta}_{2}}{2} x_{1}\right)\right]\right\}, & \text { if } x_{2} \geq x_{1} \geq 0\end{cases}
$$

where $\boldsymbol{\theta}_{i} \in(0,1]$, and $\lambda_{i}>0, i=1,2$. This distribution was obtained in [12] and can be named Generalized Gumbel's bivariate exponential distribution with parameters $\lambda_{i}$ and $\boldsymbol{\theta}_{i}, i=1,2$. If $\boldsymbol{\theta}_{1}=\boldsymbol{\theta}_{2}=\theta$, we get the Gumbel distribution considered in Example 1. The marginal survival functions are $S_{X_{i}}\left(x_{i}\right)=\exp \left\{-\lambda_{i} x_{i}\right\}$, $i=1,2$.

The survival function of the residual lifetime vector $\mathbf{X}_{t}$ is given by (8). After some algebra, we get the corresponding survival copula 


$$
C_{t}(u, v)=\left\{\begin{array}{l}
u v \exp \left\{-\frac{\boldsymbol{\theta}_{1}}{\gamma_{1}(t) \gamma_{2}(t)} \ln u \ln v\right\} \exp \left\{-\frac{\lambda_{1}\left(\boldsymbol{\theta}_{2}-\boldsymbol{\theta}_{1}\right)}{2 \lambda_{2} \gamma_{1}^{2}(t)}(\ln v)^{2}\right\} \\
\text { if } u^{-\lambda_{2} \gamma_{1}(t)} \geq v^{-\lambda_{1} \gamma_{2}(t)} \\
u v \exp \left\{-\frac{\boldsymbol{\theta}_{2}}{\gamma_{1}(t) \gamma_{2}(t)} \ln u \ln v\right\} \exp \left\{-\frac{\lambda_{2}\left(\boldsymbol{\theta}_{1}-\boldsymbol{\theta}_{2}\right)}{2 \lambda_{1} \gamma_{2}^{2}(t)}(\ln u)^{2}\right\} \\
\text { if } u^{-\lambda_{2} \gamma_{1}(t)}<v^{-\lambda_{1} \gamma_{2}(t)}
\end{array}\right.
$$

where $\gamma_{1}(t)=1+\lambda_{1} \boldsymbol{\theta}_{2} t, \gamma_{2}(t)=1+\lambda_{2} \boldsymbol{\theta}_{1} t$ and $u, v \in(0,1]$. Fix $a_{i}=\lambda_{1} \lambda_{2} \boldsymbol{\theta}_{i}$, $i=1,2$ in (12) to verify that

$$
C_{t}\left(\exp \left\{-\lambda_{1} x_{1}-\lambda_{1} \lambda_{2} \boldsymbol{\theta}_{1} x_{1} t\right\}, \exp \left\{-\lambda_{2} x_{2}-\lambda_{1} \lambda_{2} \boldsymbol{\theta}_{2} x_{2} t\right\}\right)=\frac{S\left(x_{1}+t, x_{2}+t\right)}{S(t, t)},
$$

for all $t \geq 0$. Therefore, the generalized Gumbel's bivariate exponential distribution is member of the class $\mathscr{L}(\mathbf{x} ; \mathbf{a})$.

\section{Bivariate Survival Functions with Nonaging Survival Copulas}

In this section, we will consider nonaging survival copulas $C(u, v)$ instead of memoryless dependence functions $\Omega_{t}\left(x_{1}, x_{2}\right)$.

Let us denote by $\mathscr{A}$ the class of continuous bivariate survival functions $S\left(x_{1}, x_{2}\right)$, such that $\left(X_{1}, X_{2}\right)$ and $\mathbf{X}_{t}$ have the same survival copula $C(u, v)$. Therefore, the functional equation

$$
\frac{C\left(S_{X_{1}}\left(x_{1}+t\right), S_{X_{2}}\left(x_{2}+t\right)\right)}{C\left(S_{X_{1}}(t), S_{X_{2}}(t)\right)}=C\left(\frac{C\left(S_{X_{1}}\left(x_{1}+t\right), S_{X_{2}}(t)\right)}{C\left(S_{X_{1}}(t), S_{X_{2}}(t)\right)}, \frac{C\left(S_{X_{1}}(t), S_{X_{2}}\left(x_{2}+t\right)\right)}{C\left(S_{X_{1}}(t), S_{X_{2}}(t)\right)}\right)
$$

has to be satisfied for all $x_{1}, x_{2} \geq 0$ and $t \geq 0$. We will assume further that the survival copula $C$ is time invariant (or nonaging) if it corresponds to a member of the class $\mathscr{A}$.

Taking into account the conclusion in Remark 1, all bivariate survival functions possessing BLMP belong to $\mathscr{A}$. It happens that this time invariance property is not restricted to BLMP survival functions. For instance, it is well-known that the Clayton bivariate survival function given by

$$
S\left(x_{1}, x_{2}\right)=\left[S_{X_{1}}^{-\theta}\left(x_{1}\right)+S_{X_{2}}^{-\theta}\left(x_{2}\right)-1\right]^{-1 / \theta}, \quad \theta \in(0, \infty),
$$

has time invariant survival copula. One can find other members of the class $\mathscr{A}$ in Examples 3 and 4. 
Let $\mathscr{D}(t)=\left\{(u, v) \in(0,1] \mid u=S_{X_{1}}(t), v=S_{X_{2}}(t), t>0\right\}$ be a curve on the unit square parameterized by $t>0$. In such a case, from (15) we may obtain nonaging survival copulas whenever $C$ is invariant on the curve $\mathscr{D}(t)$. In particular, if $X_{1} \stackrel{d}{=} X_{2}$, we have invariance of the survival copula along the main diagonal of the unit square.

Example 3 [Invariance on the main diagonal] The Cuadras-Augé survival copula

$$
C_{\alpha}(u, v)=[\min (u v)]^{\alpha}[u v]^{1-\alpha}, \quad \alpha \in[0,1]
$$

is invariant on the main diagonal of the unit square, see [2]. Let us initially consider equally distributed marginals $S_{X_{1}}(x)=S_{X_{2}}(x)=S_{X}(x)$. If $S_{X}(x)$ is exponentially distributed, then $S\left(x_{1}, x_{2}\right)=C_{\alpha}\left(S_{X_{1}}\left(x_{1}\right), S_{X_{2}}\left(x_{2}\right)\right)$ is a particular case of the Marshall-Olkin's bivariate exponential distribution, see [9], possessing BLMP and, consequently, belonging to the class $\mathscr{A}$. Now, let $X$ be gamma distributed random variable. In this case, BLMP does not hold true but the corresponding joint survival function still belongs to $\mathscr{A}$.

In a third scenario, where $X_{1}$ and $X_{2}$ do not share the same distribution but are joined by the Cuadras-Augé survival copula, $S\left(x_{1}, x_{2}\right)$ neither possesses BLMP nor belongs to $\mathscr{A}$.

Example 4 [Invariance along a curve] The Marshall-Olkin survival copula

$$
C_{\alpha, \beta}(u, v)=\min \left(u^{1-\alpha} v, u v^{1-\beta}\right), \quad \alpha, \beta \in(0,1)
$$

is invariant on the curve $\left\{(u, v)=\left(t^{\alpha}, t^{\beta}\right), t \in(0,1)\right\}$, see [2]. Notice that when $\alpha=\beta$ we obtain the Cuadras-Augé survival copula from Example 3.

Let us consider a baseline survival function $S_{X}(x)$ and substitute $S_{X_{1}}(x)=$ $\left[S_{X}(x)\right]^{\alpha}$ and $S_{X_{2}}(x)=\left[S_{X}(x)\right]^{\beta}$. Then, the corresponding joint survival function $S\left(x_{1}, x_{2}\right)=C_{\alpha, \beta}\left(S_{X_{1}}\left(x_{1}\right), S_{X_{2}}\left(x_{2}\right)\right)$ belongs to $\mathscr{A}$. In particular, if the marginals are exponentially distributed, not necessarily sharing the same parameter, then $S\left(x_{1}, x_{2}\right)$ possesses BLMP. But choosing $X_{1}$ exponentially distributed and $X_{2}$ beta distributed, say the corresponding joint survival function is not a member of the class $\mathscr{A}$.

The cases considered in the last two examples depend on the choice of the marginal survival functions. A general invariance property can be obtained when we consider the Clayton survival copula. In such a case, for any marginals we have time invariant survival copulas. We refer the reader to Sect. 4 in [2] for more details on time invariant copulas.

In fact, the Clayton survival copula is the only absolutely continuous copula that is preserved even under bivariate truncation, see [11]. The absolutely continuous assumption is relaxed in Theorem 4.1 in [3]. In [10], it is given a characterization of the survival functions which simultaneously have Clayton survival copula and possess BLMP, see their Theorem 3.2.

In the next statement, we establish a necessary condition to an absolutely continuous bivariate survival function be a member of the class $\mathscr{A}$. 
Theorem 3 Let $S\left(x_{1}, x_{2}\right)$ be an absolutely continuous survival function belonging to the class $\mathscr{A}$. Then, its survival copula satisfies the functional equation

$$
C(u, v)=\left[u-\frac{f_{X_{2}}(0) C^{2}(u, 1)}{a_{0}}\right] C^{1}(u, v)+\left[v-\frac{f_{X_{1}}(0) C^{1}(1, v)}{a_{0}}\right] C^{2}(u, v),
$$

for all $u, v \in[0,1]$ and $a_{0}>0$, where $C^{1}$ and $C^{2}$ denote the partial derivatives of $C$ with respect to the first and second arguments, respectively.

Proof Take the derivative in (15) with respect to $t$ and substitute $t=0$ to get (16).

The knowledge of the first partial derivatives of the survival copula $C(u, v)$ is sufficient to recover the distribution of $\min (U, V)$, where $U$ and $V$ are uniformly distributed with survival copula $C(u, v)$. Really, $P(\min (U, V)>t)=C(t, t)$ for $t \in[0,1]$. Now, substitute $u=v=t$ in (16) to get the corresponding equation (and main diagonal copula).

Finally, we show two known functional equations which are particular cases of (16). Under assumptions of Theorem 3 , let $f_{X_{1}}(0)=f_{X_{2}}(0)$. Then

$$
C(u, v)=\left[u-\frac{C^{2}(u, 1)}{2}\right] C^{1}(u, v)+\left[v-\frac{C^{1}(1, v)}{2}\right] C^{2}(u, v) .
$$

The same equation is obtained in Proposition 3 (ii) in [1] under the condition that $X_{1}$ and $X_{2}$ are uniformly distributed on the unit square, i.e., $f_{X_{1}}(0)=f_{X_{2}}(0)=1$.

Further, assume that $C(u, v)$ is exchangeable. Thus, $C^{2}(u, 1)=C^{1}(1, u)$, $C^{2}(u, v)=C^{1}(v, u)$ and the last equation transforms into

$$
C(u, v)=\left[u-\frac{C^{1}(1, u)}{2}\right] C^{1}(u, v)+\left[v-\frac{C^{1}(1, v)}{2}\right] C^{1}(v, u),
$$

see Proposition 3 on page 18 in [4].

\section{Conclusions}

The time invariance of the residual lifetime vector $\mathbf{X}_{t}$ of $\left(X_{1}, X_{2}\right)$ is characterized by BLMP in [9]. It tells us that the joint distributions of $\mathbf{X}_{t}$ and $\left(X_{1}, X_{2}\right)$ coincide independently of $t$, i.e., the BLMP holds. In this paper, we consider a more general concept, namely time invariance of the dependence functions of $\mathbf{X}_{t}$ and $\left(X_{1}, X_{2}\right)$, given by (4) and (1), respectively.

We offer copula representations for the time invariance property related to bivariate survival functions of the residual lifetime vector $\mathbf{X}_{t}$. While in Sect. 2, the nonaging phenomena is associated with the dependence function $\Omega_{t}\left(x_{1}, x_{2}\right)$, in Sect. 3 our interest is on the survival copula $C_{t}(u, v)$ of $\mathbf{X}_{t}$.

We are thankful to the referee and editor for their comments. 
Open Access This chapter is distributed under the terms of the Creative Commons Attribution Noncommercial License, which permits any noncommercial use, distribution, and reproduction in any medium, provided the original author(s) and source are credited.

\section{References}

1. Charpentier, A.: Tail distribution and dependence measures. Working paper (2003)

2. Charpentier, A., Juri, A.: Limiting dependence structures for tail events, with applications to credit derivatives. J. Appl. Probab. 43, 563-586 (2006)

3. Durante, F., Jaworski, P.: Invariant dependence structure under univariate truncation. Stat: J. Theoret. Appl. Stat. 46, 263-277 (2012)

4. Gourieroux, C., Monfort, A.: Age and term structure in duration models. Working paper (2003)

5. Gumbel, E.: Bivariate exponential distributions. J. Am. Stat. Assoc. 55, 698-707 (1960)

6. Hofert, M., Vrins, F.: Sibuya copulas. J. Multivar. Anal. 114, 318-337 (2013)

7. Kulkarni, H.: Characterizations and modelling of multivariate lack of memory property. Metrika 64, 167-180 (2006)

8. Mai, J.-F., Scherer, M.: Simulating Copulas. Imperial College Press, London (2012)

9. Marshall, A., Olkin, I.: A multivariate exponential distribution. J. Am. Stat. Assoc. 62, 30-41 (1967)

10. Mulero, J., Pellerey, F.: Bivariate aging properties under Archimedean dependence structures. Commun. Stat: Theor. Methods. 39, 3108-3121 (2010)

11. Oakes, D.: On the preservation of copula structure under truncation. The Canadian J. Stat. 33, 465-468 (2005)

12. Pinto, J.: Deepening the notions of dependence and aging in bivariate probability distributions. PhD Thesis, University of Sao Paulo (2014)

13. Sibuya, M.: Bivariate extreme statistics I. Ann. Inst. Stat. Math. 11, 195-210 (1960) 